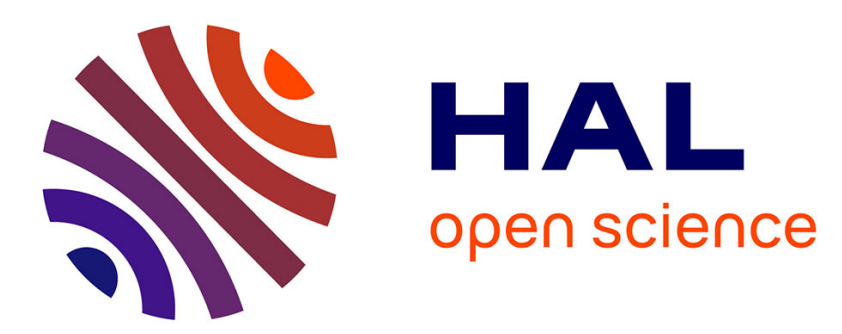

\title{
Smart Route Request for on-demand route discovery in constrained environments
}

\author{
Jiazi Yi, Thomas Heide Clausen, Antonin Bas
}

\section{To cite this version:}

Jiazi Yi, Thomas Heide Clausen, Antonin Bas. Smart Route Request for on-demand route discovery in constrained environments. 2012 IEEE International Conference on Wireless Information Technology and Systems (ICWITS), Nov 2012, Maui, United States. pp.1-4, 10.1109/ICWITS.2012.6417755 . hal-02263397

\section{HAL Id: hal-02263397 \\ https://hal-polytechnique.archives-ouvertes.fr/hal-02263397}

Submitted on 4 Aug 2019

HAL is a multi-disciplinary open access archive for the deposit and dissemination of scientific research documents, whether they are published or not. The documents may come from teaching and research institutions in France or abroad, or from public or private research centers.
L'archive ouverte pluridisciplinaire HAL, est destinée au dépôt et à la diffusion de documents scientifiques de niveau recherche, publiés ou non, émanant des établissements d'enseignement et de recherche français ou étrangers, des laboratoires publics ou privés. 


\title{
Smart Route Request for On-demand Route Discovery in Constrained Environments
}

\author{
Jiazi Yi, Thomas Clausen, Antonin Bas \\ Laboratoire d'Informatique (LIX) - Ecole Polytechnique, France \\ jiazi@jiaziyi.com, Thomas@ThomasClausen.org, antonin@antonin-bas.fr
}

\begin{abstract}
A derivative of AODV, denoted LOADng, is proposed for use in very constrained environment, sacrificing a number of features from AODV for the benefit of smaller control messages and simpler processing logic. Among these sacrifices is intermediate route replies. This paper presents an alternative to intermediate router replies, denoted Smart Route Request, which provides an optimization similar to that attainable by intermediate route requests, but without imposing additional processing complexity or additional signaling. A performance study is presented, showing that the use of Smart Route Requests can effectively reduce the control traffic overhead from Route Requests, while retaining the simplicity of LOADng. LOADng with Smart Route Requests effectively reduces control traffic overhead and on-link traffic collisions, and this especially for multipoint-to-point traffic.
\end{abstract}

\section{INTRODUCTION}

Since the late $90 \mathrm{~s}$, the $\mathrm{IETF}^{1}$ has embarked upon a path of developing routing protocols for networks with increasingly more fragile and low-capacity links, with less pre-determined connectivity properties and with increasingly constrained router resources. In '97, by chartering the MANET working group, then subsequently in 2006 and 2008 by chartering the 6LowPAN and ROLL working groups.

The MANET working group converged on the development of two protocol families: reactive protocols, including AODV [1], and proactive protocols, including Optimized Link State Routing (OLSR) [2]. A distance vector protocol, AODV operates in an on-demand fashion, acquiring and maintaining routes only while needed for carrying data, by way of a Route Request-Route Reply exchange.

After acquiring operational experiences, the MANET working group commenced developing successors to OLSR and AODV, denoted OLSRv2 and DYMO. Whereas a relatively large and active community around OLSR thus standardized OLSRv2 [3], [4], [5], [6] and [7], the momentum behind DYMO withered in the MANET working group ${ }^{2}$. However, other derivatives of AODV have been implemented and used widely, such as IEEE 802.11s [8], and the G3-PLC standard [9], published in 2011, specifies the use of LOAD [10] (a simplified version of AODV) at the MAC layer, for providing mesh-under routing for utility (electricity) metering networks. Spurred by these experiences, 2011 saw the emergence of LOADng [11], as a successor to LOAD.

\section{A. Statement of Purpose}

For on-demand routing protocols, like AODV and its derivatives (LOAD, LOADng), the route discovery process is the most costly operation in terms of control traffic overhead: a Route Request (RREQ) message is flooded through the whole network, with the sought destination replying by unicasting a Route Reply (RREP). In order to reduce the RREQ flooding overhead, AODV makes use of intermediate RREPs: a router, which is not the sought destination but which has a valid route to the destination, does not re-flood the RREP but rather transmits in unicast an intermediate RREP to the source, and a gratuitous RREP to the sought destination. Thus, AODV makes use of existing routing information present in the network to "cut short" the RREQ flooding process - at the expense of more sequence numbers in control packets (and more complex processing) in order to avoid generating loops. LOAD and LOADng eliminate intermediate RREP, and permit only the sought destination to reply to a RREQ, so as to prevent loops - with the motivation being that in constrained environments, it is necessary to be draconian in reducing the control message sizethe state that a router needs to maintain, and to and the complexity of protocol operation. Alas, it is evident that in certain scenarios, this elimination to not existing routing information can cause more control messages to be required for protocol operation.

This paper presents a simple mechanism, SmartRREQ, whereby the benefits of intermediate RREPs as known from AODV are enabled, whilst avoiding the inconveniences of larger control messages and additional state and processing complexity.

\section{B. Paper Outline}

The remainder of this paper is organized as follows: section II briefly introduces LOADng, by way of emphasizing the differences with AODV. Section III discusses intermediate RREQs, and introduces SmartRREQ. Section IV presents a performance evaluation of LOADng with and without the use of SmartRREQ, and finally section V concludes this paper.

\footnotetext{
${ }^{1}$ http://www.ietf.org/

${ }^{2} \mathrm{http}: / /$ tools.ietf.org/wg/manet/minutes?item=minutes $81 . \mathrm{html}$
} 


\section{ON-DEMAND ROUTING IN CONSTRAINED ENVIRONMENT: LOADNG}

A lightweight derivative of AODV, targeted for use in low power and constrained environments, LOADng [11] inherits the basic properties and operations of AODV: generation of Route Requests (RREQs) by a router (originator) for discovering a route to a destination, forwarding of such RREQs until they reach the destination router, generation of Route Replies (RREPs) upon receipt of an RREQ by the indicated destination, and unicast hop-by-hop forwarding of these RREPs towards the originator. If a route is detected broken, i.e., if the forwarding of a data packet to the recorded next hop on the route to the destination is detected to fail, local route repair can be attempted, or a Route Error (RERR) message can be returned to the originator of that data packet. Compared to AODV, LOADng is both simplified and extended, as summarized in the following.

Compared to AODV, LOADng is extended to:

- Be modular, with a core specification defining simple and light-weight core functions of the protocol.

- Support optimized flooding of RREQs for control traffic reduction, and employs jitter to reduce the probability of losses due to collisions on lower layers [3].

- Support address lengths from 1-16 octets ${ }^{3}$. The only requirement is, that within a given routing domain, all addresses are of the same address length.

- Supports different routing metrics. Different metrics are supported, to make better use of link information from different layers.

Compared to AODV, LOADng is simplified to:

- permit only the destination to respond to an RREQ; intermediate routers are explicitly prohibited from responding to RREQs, even if they may have active routes to the sought destination. All messages (RREQs or RREPs) generated by a given router share a single unique, monotonically increasing sequence number. This also eliminates Gratuitous RREPs while ensuring loop freedom. The rationale for this simplification is reduced complexity of protocol operation and reduced message sizes - found to be without significant influence in the performance.

- not maintain a precursor list, thus when forwarding of a data packet to the recorded next hop on the route to the destination fails, an RERR is sent only to the originator of that data packet. The rationale for this simplification is an assumption that few overlapping routes are in use concurrently, and delay is not a critical issue in a resource-constrained network.

\section{EFFICIEnt Route Discovery AND SMART Route REQuest}

Reducing the overhead, delay and complexity of the route discovery process (RREQ/RREP exchange) is key to adapting on-demand routing protocols for use in constrained environments. In this section, the route discovery processes used in AODV and LOAD/LOADng are described, and the SmartRREQ mechanism is proposed.

\section{A. Intermediate Route Replies: to be, or not to be.....}

During the Route Discovery process of AODV, an intermediate router can generate an intermediate RREP in response to an RREQ, if it has a valid route to the destination sought - and must also generate a gratuitous RREP and send this to the desired destination in order to establish a complete and bi-directional route. In order to avoid routing loops when permitting intermediate routers to generate intermediate RREPs, an RREQ in AODV carries an RREQ ID, destination sequence number, and originator sequence number in RREQ messages - recorded and maintained by intermediate routers, and used for when processing RREQs and RREPs.

In LOADng, intermediate RREPs are prohibited, so as to reduce the control message size and guarantee loop freedom. A router, receiving an RREQ, is either the ultimate destination - and, if so, must respond by an RREP - or is an intermediate router - and, if so, has to rebroadcast the RREQ, even if it otherwise has a valid route to the destination. [12] shows that LOADng is more adapted to constrained environments with lower routing overhead and less collisions.

While eliminating intermediate and gratuitous RREPs reduce the size of RREQ/RREPs, it might result in more RREQ (re-)transmissions. Consider the obvious case where a set of routers in the same part of the network topology, for example, all seek a route to a gateway: not using the topology information in intermediate routers will cause all RREQs to have to transverse the network, and RREPs to be sent back.

In some network types, such as sensor networks, it is common to have sensor-to-root (multipoint-to-point - or MP2P) traffic. While eliminating intermediate RREP can reduce the size of control message and simplify the protocol process, the side effect of blindly flooding RREQ cannot be ignored in this kind of scenarios.

\footnotetext{
${ }^{3}$ i.e., IPv6, IPv4, 6LowPAN short addresses, L2 MAC addresses etc. are all supported by LOADng
} 


\section{B. Smart Route Request}

To avoid blindly flooding RREQ in scenarios where MP2P traffic prevails, SmartRREQs is proposed. Retaining the lightweight nature of LOADng, and incurring no additional signaling, SmartRREQ takes benefits of existing routing information in intermediate routers.

On receiving an RREQ message, an intermediate router (neither source nor destination) performs the following procedure:

1) If the intermediate router has a valid route to the destination, AND the $<$ next-hop $>$ field of the corresponding routing tuple is not equal to the previous hop address of the RREQ, then the RREQ is unicast to the $<$ next-hop $>$.

2) Otherwise the RREQ is broadcast as usual to all its neighbors.

This is illustrated in figure 1: $S$ solicitates a route to $D$. $A$ and $B$ already have routes to $D$, and upon receiving the RREQ initiated by $S$ will unicast the RREQ, according to their routing table. When the RREQ arrives the destination, the RREP is unicast as to the source.

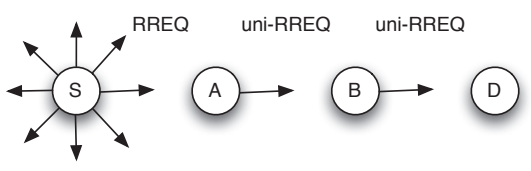

Figure 1. LOADng Route Discovery with smart RREQ. $S$ initiates an RREQ for $D . A$ and $B$ already have an available route to $D$

With this, in some scenarios such as MP2P networks, in which the neighbor routers have already available routes to the destination, an RREQ message will stay local, rather than being flooded to the whole network. The RREQs will be unicast to the destination only. If an intermediate router detects a broken link when trying to send an unicast RREQ, then it should broadcast the RREQ instead.

\section{Simulation And Evaluation}

The proposed SmartRREQ is implemented, studied and evaluated by way of network simulations using NS2. Simulations were made with from 50 to 500 routers, using IEEE 802.11 wireless interfaces, and placed statically and randomly in a square field of varying size.

The network is subject to point-to-point (P2P) traffic or multipoint-to-point (MP2P) traffic with routers generating 80 second bursts of 512-octet data packets every 5 seconds. The simulation results in different scenarios comparing LOADng with or without SmartRREQ, as well as comparing to $\mathrm{AODV}^{4}$ are be presented in this section.

\section{A. Point-to-Point Traffic}

For the purpose of studying P2P traffic performance, 50 to 100 routers were distributed randomly in a field of $1000 \mathrm{~m} \times 1000 \mathrm{~m}$. The network was subject to 30 concurrent and random (source, destination) traffic flows. Given the low traffic load in these scenarios, LOADng, LOADng with Smart RREQ and AODV all attain identical packet delivery ratios of almost $100 \%$.

Figures 2(a) and 2(b) show the number of collisions and the average control traffic overhead measured in number of bytes sent over the network, respectively. A first observation is, that LOADng transmits much fewer bytes across the network than AODV, and that using SmartRREQ reduces this overhead further. Additionally, LOADng yields fewer collisions than does AODV, and fewer still when using also SmartRREQ due to less control packets being flooded through the networks. The increased overhead and collisions from AODV is, in part, due to larger messages and in part due to AODVs use of expanding ring flooding.

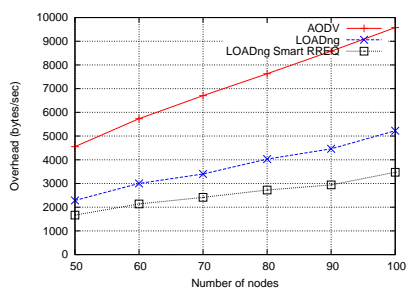

(a) Average control traffic overhead

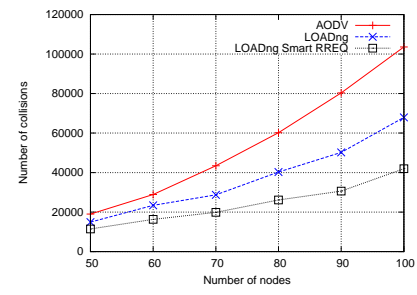

(b) Number of collisions

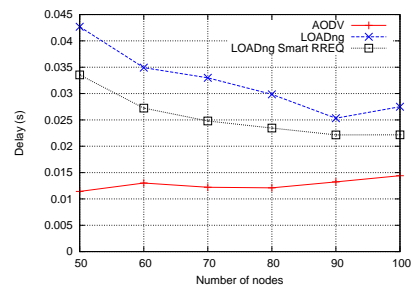

(c) Average end-to-end delay

Figure 2. Simulation results of P2P scenarios

The average end-to-end delay is shown in figure 2(c). Compared to LOADng, using SmartRREQ yields a lower delay, due to the reduced number of collisions (which can result in more MAC layer retransmission). Alas, the delays are not quite as

\footnotetext{
${ }^{4}$ With Expanding Ring and intermediate/gratuitous RREPs.
} 
low as that attained by AODV: when using SmartRREQ, even if an intermediate router has a valid route to the destination, an RREQ will have travel (albeit in unicast) to the ultimate destination and an RREP returned, whereas for AODV an intermediate RREP is generated and immediately sent back from the intermediate router. Another reason of the longer delay of LOADng is because jitter [3] is used, which introduces random delay at each transmission of RREQ message to reduce collision.

\section{B. Multipoint-to-Point Traffic}

For MP2P scenarios, larger networks and higher traffic loads are tested. The network has from 50-500 routers, with a single fixed router designated as the "root" - to which all the other routers send a data packet of 512-octet every 5 seconds. For all scenarios, the same router density is maintained, i.e.,, as the number of routers increase, so does the network field size.

The packet delivery ratio is shown in figure 3(a), showing that while the LOADng delivery ratio drops significantly , to as low as about $20 \%$ in a 500 router big network, using SmartRREQ and AODV, delivery ratios of $100 \%$ are attained.

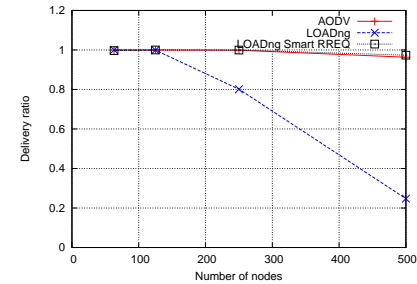

(a) Delivery ratio

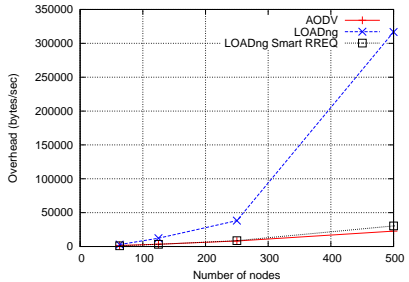

(b) Average overhead

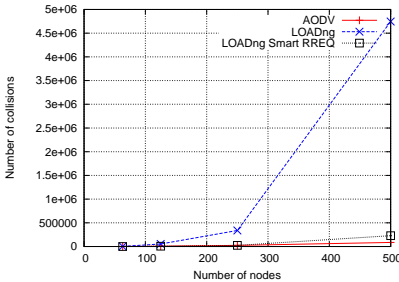

(c) Number of collisions

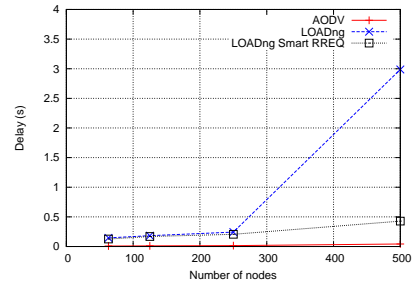

(d) Average end-to-end delay

Figure 3. Simulation results of MP2P scenarios

In fact, in MP2P scenarios, since every router has to maintain a route to the root, when one router initiates an RREQ message, its neighbors have a high probability of still having an active route to the root (as illustrated in figure 1). Thus, a network-wide RREQ flooding can be avoided, and the routing overhead significantly reduced. Due to the the high overhead and collision rate of blind RREQ flooding, LOADng also incurs also higher delays, as illustrated in figure 3(d).

\section{CONCLUSION}

The proposed on-demand routing protocol for constrained environments, LOADng, sacrifices the intermediate/gratuitous RREPs of AODV, in order to provide a simpler protocol with smaller control messages, less state and complexity - and while still ensuring loop freedom. Alas, for some common scenarios, such as data acquisition networks, wherein all traffic terminates at a central collection point, this sacrifice is also at the expense of performance.

This paper proposes SmartRREQ as an alternative to the intermediate/gratuitous RREP of AODV. Maintaining the same mode of operation as LOADng, wherein only the ultimate destination for an RREQ is allowed to generate an RREP, SmartRREQ allows an intermediate router, which has a path to the sought destination, to retransmit a received RREQ in unicast to that destination, thereby eliminating flooding when possible. This requires no extra signaling or overhead, nor any extra processing, as compared to LOADng.

This mechanism has been tested by way of network simulations, for which it is worth observing, that for all tested scenarios, LOADng with SmartRREQ attains the same data delivery ratios as does AODV, with identical or lower overheads (and less router state and simpler processing). AODV does, however, yield slightly lower delays. Compared to LOADng without SmartRREQ, significant improvements are obtained, according to all metrics studied. This allows the conclusion that SmartRREQ is an indispensable component for use in LOADng - yielding all gains and no griefs.

\section{REFERENCES}

[1] C. Perkins, E. Belding-Royer, and S. Das, "Ad hoc On-Demand Distance Vector (AODV) Routing," Experimental RFC 3561 , July 2003.

[2] T. Clausen and P. Jacquet, "Optimized Link State Routing Protocol (OLSR)," Experimental RFC 3626, October 2003.

[3] T. Clausen, C. Dearlove, and B. Adamson, "Jitter Considerations in MANETs," IETF Inf. RFC 5148, February 2008.

[4] T. Clausen, C. Dearlove, J. Dean, and C. Adjih, "Generalized MANET Packet/Message Format," Std. Track RFC 5444, February 2009.

[5] T. Clausen and C. Dearlove, "Representing Multi-Value Time in MANETs," IETF Std. Track RFC 5497, February 2009.

[6] T. Clausen, C. Dearlove, and J. Dean, "Mobile Ad Hoc Network Neighborhood Discovery Protocol," Std. Track RFC 6130, April 2010

[7] T. Clausen, C. Dearlove, and P. Jacquet, "The Optimized Link State Routing Protocol version 2," Internet Draft, draft-ietf-manet-olsrv2-11, work in progress, April 2010.

[8] G. Hiertz, S. Max, R. Zhao, D. Denteneer, and L. Berlemann, "Principles of ieee 802.11s," in Proceedings of WiMAN in conjunction with the 16th ICCCN, Honolulu, Hawaii, USA, Aug 2007, p. 6.

[9] "ITU-T G.9956: Narrow-Band OFDM power line communication transceivers - Data link layer specification," November 2011.

[10] K. Kim, S. D. Park, G. Montenegro, S. Yoo, and N. Kushalnagar, “6LoWPAN Ad Hoc On-Demand Distance Vector Routing," June 2007, Internet Draft, work in progress, draft-daniel-6lowpan-load-adhoc-routing-03.

[11] T. Clausen, A. C. de Verdiere, J. Yi, A. Niktash, Y. Igarashi, H. Satoh, and U. Herberg, "The lln on-demand ad hoc distance-vector routing protocol next generation," The Internet Engineering Task Force, October 2011, Internet Draft, work in progress, draft-clausen-lln-loadng.

[12] T. Clausen, J. Yi, and A. C. de Verdiere, "Loadng: Towards aodv version 2." Proceedings of IEEE 76th Vehicular Technology Conference: VTC2012-Fall, 2012. 